\title{
Nanotechnology Research for Food Science in Japan
}

\author{
Nakajima, M. \\ Food Engineering Division, National Food Research Institute, NARO \\ 2-1-12, Kannondai, Tsukuba, Ibaraki 305-8642, Japan, *E-mail: mnaka@affrc.go.jp
}

\begin{abstract}
Nanotechnology is one of the most important research fields among life sciences, information and telecommunications, environmental sciences, and nanotechnology/materials which are selected by Japanese Government. Ministry of Agriculture, Forestry and Fisheries (MAFF) has carried out 5-year project "Development of Nanotechnology and Materials for Innovative Utilizations of Biological Functions" since 2002. The project is composed of 6 research teams: "Development of nano-structured tissue culture plates"; "Development of process technology for monodisperse nanoparticles and their utilization such as in a drug delivery system"; "Development of new functional biomaterials by controlling molecular orientation and so forth"; "Development of technologies for nanoscale analysis and modification of biomolecules"; "Evaluation and utilization of dynamic states of water cluster" and "Development of micro-bioreactor system". This paper will present the overview of the nanotechnology project for food and agriculture sciences in Japan.
\end{abstract}

Key words: Nanotechnology, Monodisperese emulsion, Nano-structured tissue culture, Nanoscale analysis, Micro-bioreactor system

\section{Introduction}

Nanotechnology is an interdisciplinary science and technology. In the past research projects in the Ministry of Agriculture, Forestry and Fisheries (MAFF) of Japan, biological materials and their functions were studied, therefore it would be good to explore new field by utilizing the accumulated knowledge and experience and collaborating with other nanotechnology and engineering fields. Actually, some researchers in MAFF have been already involved in nanotechnology, precisely microtechnology. By taking into account the situation, Agriculture, Forestry and Fisheries Research Council Secretariat (AFFRC), MAFF, planned a 5-year project "Development of Nanotechnology and Materials for Innovative Utilizations of Biological Functions" with the one-year budget of about 150-200 million yen since 2002. The project is composed of 6 teams: "Development of nano-structured tissue culture plates"; "Development of process technology for monodisperse nanoparticles and their utilization such as in a drug delivery system"; "Development of new functional biomaterials by controlling molecular orientation and so forth"; "Development of technologies for nanoscale analysis and modification of biomolecules"; "Evaluation and utilization of dynamic 
states of water cluster" and "Development of microbioreactor system". The second author of this paper was involved in the start up of the Project at AFFRC, presented on this nanotechnology project at the United States - Japan Cooperative Program in Natural Resources (UJNR), Food and Agriculture Panel meeting [Yamamoto, 2002]. National Food Research Institute (NFRI) and AFFRC organized the symposium on this Project and the Proceedings are published [Nakajima, 2004]. This paper describes the development of the project, and also some recent results.

\section{Development of nano-structured tissue culture plates}

Kikuchi (formerly NFRI) has developed a new technology for blood rheology using microfabricated silicon channel array by semiconductor technology. From determining the passing time of $100 \mu \mathrm{L}$ human blood through $5 \mu \mathrm{m}$ sized microchannel array which is blood capillary model, investigations on health and blood rheology have been done [Kikuchi et al, 2001]. Figure 1 shows healthy blood flows through microchannel and clotted on the plate. The microchannel array fluid analysis equipment, which is called MCFan is now in commercially available in Japan. Based on this technology, Kikuchi and Yazawa are exploiting this microchannel technology for tissue cell culture system, and scheme is shown in Figure 2 [Yamamoto, 2002].
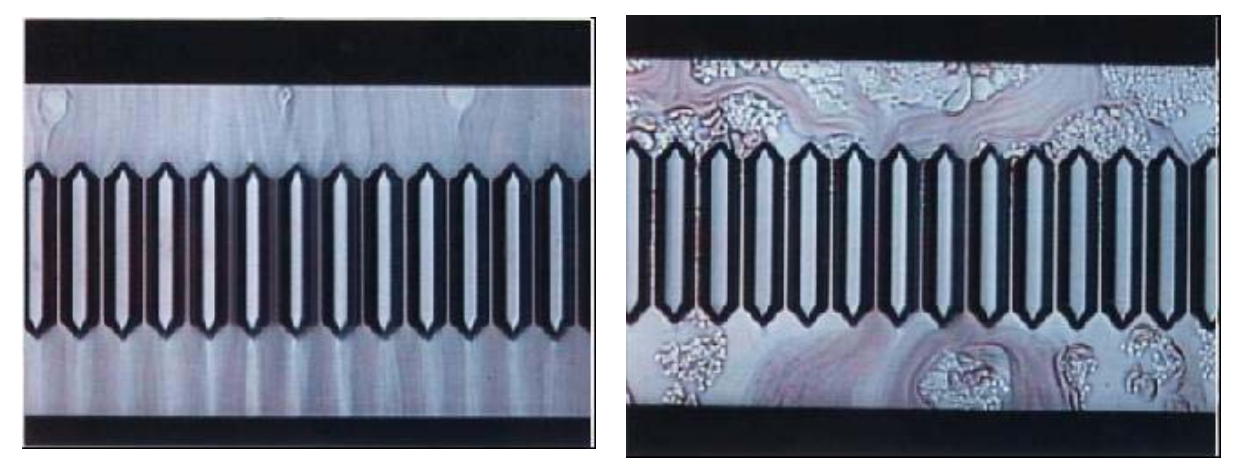

Fig. 1. Healthy blood flows through microfabricated channel arrays and blood-clotted on the microchannel terrace

As it is pointed out by many researchers, trial and error are always required to establish a new tissue cell culture system, and it is difficult to maintain the tissue culture system in a stable state for a long time. To overcome the difficulty, micro-pit tissue culture plate was invented [Yazawa and Kikuchi, 2004]. On a silicon plate several pits in micro-meter level in area and depth were processed by microprocessing technology. It was found out that osteoblasts grew only at the bottom of the pits and fibroblasts grew only on the rims of the pits. The growth rates of both tissue cells were higher than those in the conventional tissue culture systems. In the project, different structures of silicon and polymer microchannels are 
designed and used experimentally, and the micro-pit will be optimized for the other tissue cells. Recently Kuraray Co. has developed disposable polymer micro-pit tissue plates as shown in Figure 3 with the collaboration of NFRI, and the disposable plates have been investigated for medical utilization [Kuraray, 2006].

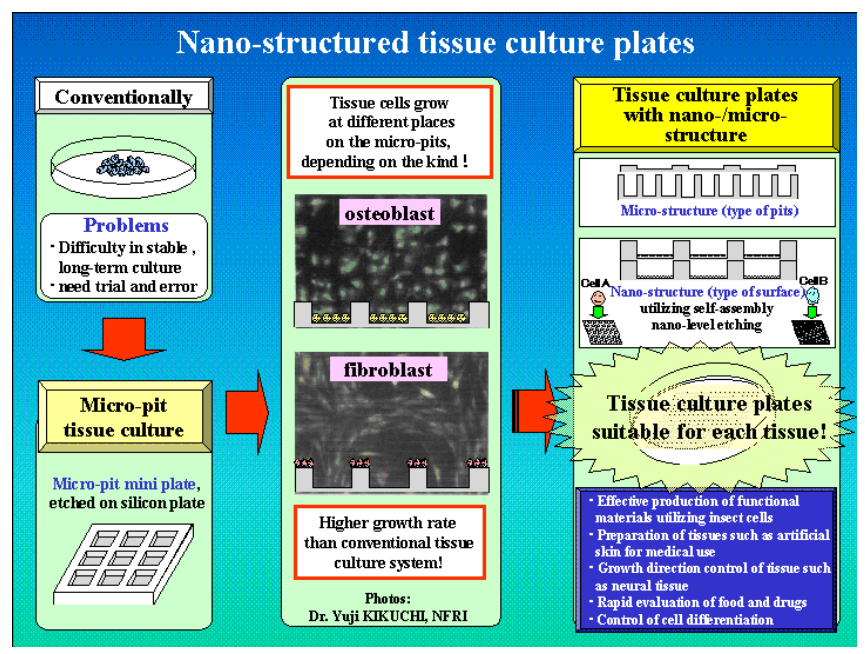

Fig. 2. Development of nano-structured tissue culture plates.
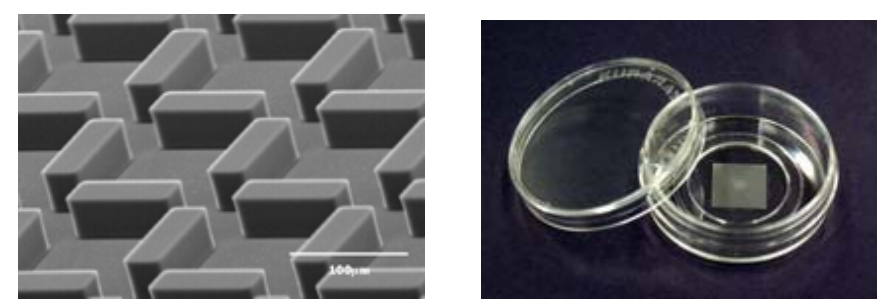

Fig. 3. Developed polymer tissue culture plates based on the Project

\section{Development of process technology for monodisperse nanoparticles and their utilization such as in a drug delivery system}

Emulsions are widely used in food, pharmaceutical and chemical industries. Conventional mechanical emulsification equipments such as collide mill and high pressure homogenizer are commonly used, however, obtained emulsion droplets are polydisperse. Monosized droplets in emulsions have advantages for control of their physical and functional properties, stability, and applications to other processes. Recently we have developed microchannel (MC) technology for monosized droplets formation [Kobayashi et al, 2002; Kobayashi et al, 2005; Nakajima, 2004]. First, MC arrays with various shapes and sizes were micro-fabricated using a single-crystal silicon substrate. An emulsion was prepared by simply forcing dispersed phase fluid into continuous phase fluid through the MC. Real-time optical observations of the droplet formation process can be realized with a microscope video system. The grooved type MC and module were used to characterize MC emulsification, and 
straight-through MC and module were developed for higher productivity. Oil-in-water emulsions can be obtained due to its hydrophilic surface property after MC oxidation. Hydrophobic MC modified by silanization can be also applicable to W/O-emulsions. Both monodisperse oil-in-water and water-in-oil emulsions with average droplets sizes ranging 5-100 $\mu \mathrm{m}$ and coefficients of variations lower than $10 \%$ were successfully obtained. Emulsification was significantly affected by MC sizes and structures. Analysis by high-speed camera revealed that the structure of terrace is so important for instability at the outlet, and spontaneous droplet formation caused by interfacial tension. An oblong straight-through MC was highly applicable to emulsification [Kobayashi et al, 2002]. Figure 4 and 5 show photomicrograph of oblong straight-through $\mathrm{MC}$ and produced oil-in-water emulsion droplets. MC emulsification was also applied to productions of solid-lipid and polymeric microspheres, and microcapsules for bioprocesses.
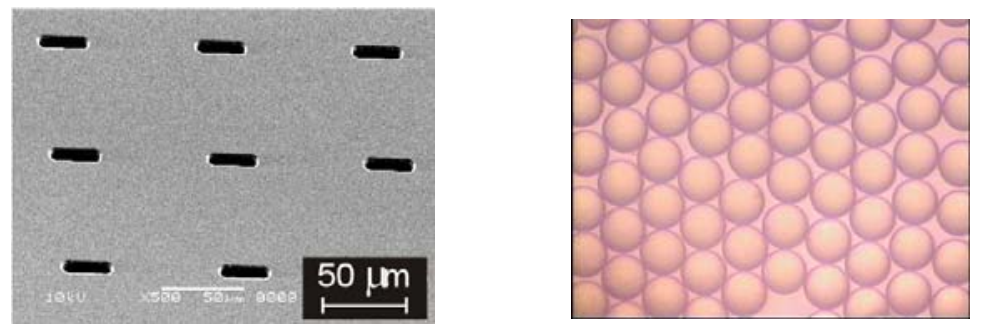

Fig. 4. Photomicrograph of oblong straight-through microchannel (MC) (left), and produced oil-in-water emulsion droplets by MC system (right)
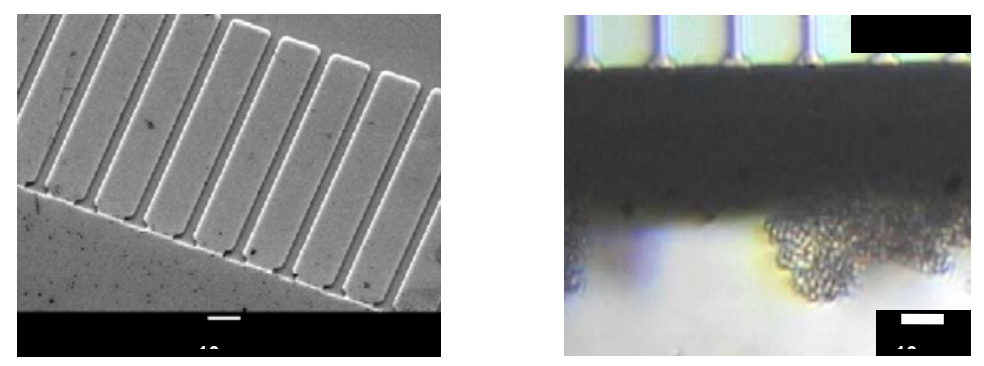

Fig. 6. Down-sized MC plate and the micrograph of droplet formation

Recently EPTEC Co. Ltd. has industrialized above NFRI patented microchannel emulsification equipment. More than 20 equipments have been already sold to some academia and chemical industries, in which they are investigating to produce monosized polymer microparticles. For smaller microparticles, channel size of plate type $\mathrm{MC}$ was reduced to 0.2-0.5 $\mu \mathrm{m}$, and about $1 \mu \mathrm{m}$-size droplets were formed shown in Figure 6. Smaller sized straight-through MC plates are currently produced with the help of MEMS and Packaging Group of Advanced Manufacturing Institute, National Institute of Advanced Industrial Science and Technology (AIST). Another microchannel type, microfabricated airflow nozzle has been successfully applied to microencapsulation of living cells into $150 \mu \mathrm{m}$ microcapsules [Sugiura et al, 2006]. 
Not only microparticles but also nanoparticles are very important for various industries, such as medical, food, cosmetic and chemical industries. In the team, downsizing the microchannels for smaller particles, development of nanoparticles formation methods and their characterization as well as the use of nanoparticle for allergen controlled food systems have been studied. The scheme is shown in Figure 7. The author of this paper is leading the team, and reported several publications, in which lipid nanoparticles are formulated and characterized [Tan and Nakajima, 2005].

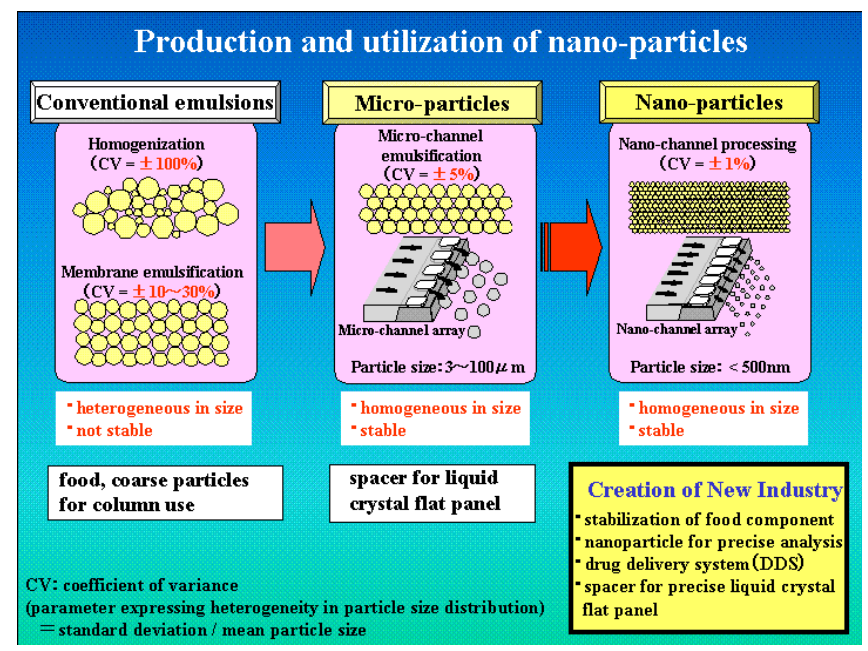

Fig. 7. Development of process technology for monodisperse nanoparticles and their utilization such as in a drug delivery system.

\section{Development of new functional biomaterials by controlling orientation and so forth}

Cellulose is the largest biomass on the earth, therefore its effective utilization in recycling society will become more important in the future. Bacterial cellulose is considered to be a promising nanofiber. Kondo has discovered nanometer-sized rail made of cellulose using acetobacter xylinum, as shown in Figure 8. Not only two-dimensional micro-structured cellulose architecture but also three-dimensional one can be possible [Kondo et al, 2002; Kondo, 2006]. From this technique, recyclable cellulose-based new materials that could replace conventional synthetic polymers have been produced [Kondo, 2004]. The scheme is shown in Figure 9.

Silk fibroin is a promising protein that is highly biocompatible. Sulphated fibroin also has an anti-HIV activity. By chemical modification and control of molecular orientation, new biocompatible material has been also developed by collaboration between chemists and medical doctors in the team. 


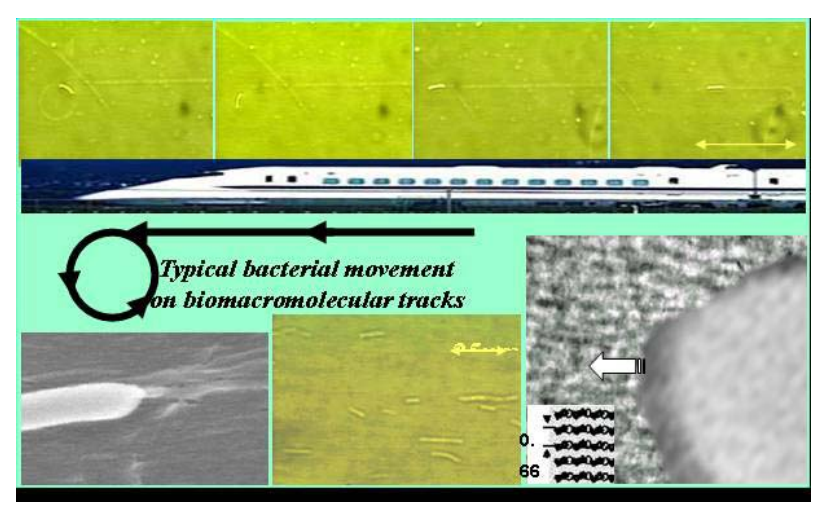

Fig. 8. Bottom-up autofabrication of 3-dimensional structures on a 2-dimensional patterned sheet using regulated Acetobacter machinery [Kondo, 2004]

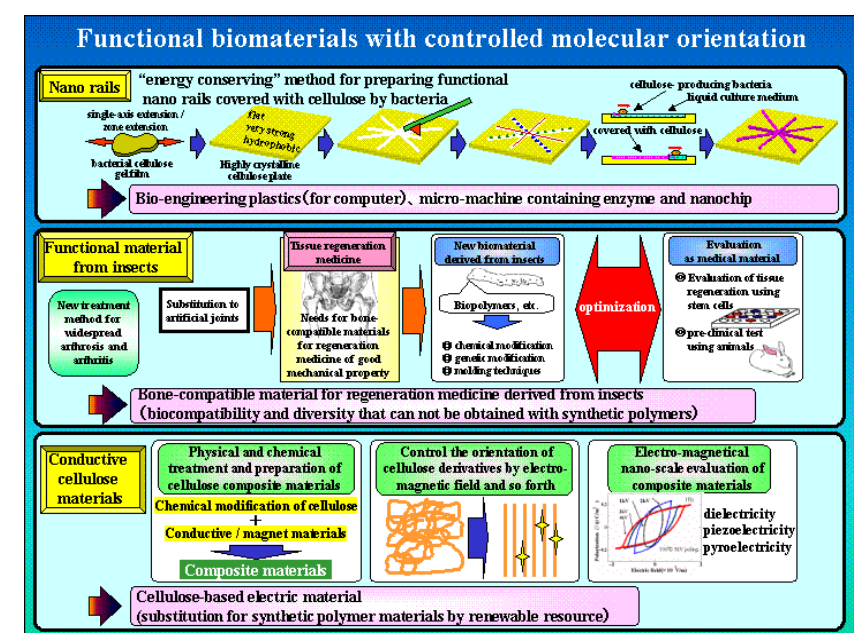

Fig. 9. Development of new functional biomaterials by controlling molecular orientation and so forth.

\section{Development of technologies for nanoscale analysis and modification of biomolecules}

Sensing proteins of insects and pheromone receptor proteins of bacteria can be used as sensor devices. By utilizing not only these specific proteins but also the other ligand-specific molecules such as ionophores and antibodies, nano-sensor could be developed. In the team, signals of interactions between ligands and their receptors will be converted into electrical signal, and sensitivity and specificity of the proteins will be improved for intensive nano-sensor use [Tamada, 2004]. In the future, integrated nano-sensor connected with a PC or a cellular phone would be able to measure many compounds with high sensitivity simultaneously, if nanotechnology reduces the size of integrated sensor devices and offers cheap sensor chips. Development of analysis and manipulation technology for bio-molecule structure is studied as shown in Figure 10 [Tamada, 2004]. 


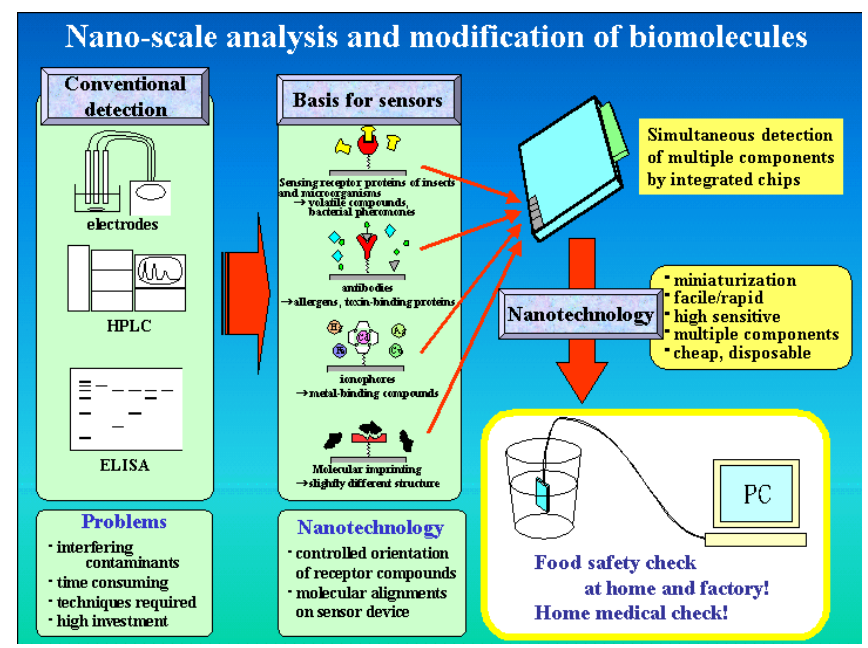

Fig. 10. Development of technologies for nano-scale analysis and modification of biomolecules.

\section{Evaluation and utilization of dynamic states of water clusters}

Water cluster has been investigated by many researchers in various fields such as physics, chemistry, and biology, however, the structure and function are still not yet clear. Water is indispensable in agriculture, forestry and fisheries, therefore, the water research is added as one of the nanotechnology project teams, and Nakanishi was nominated as the team leader. Prof. Nakanishi studies water movement in living plants by neutron beam analysis. As well as water role in living cells, water role in food processing is important. For example, super-heated vapor has been paid great attention because of its unique properties. Heating process by super-heated vapor is already realized in food industry. In the team, functionality of waters has been investigated on the viewpoint of water cluster [Nakanishi et al, 2004].

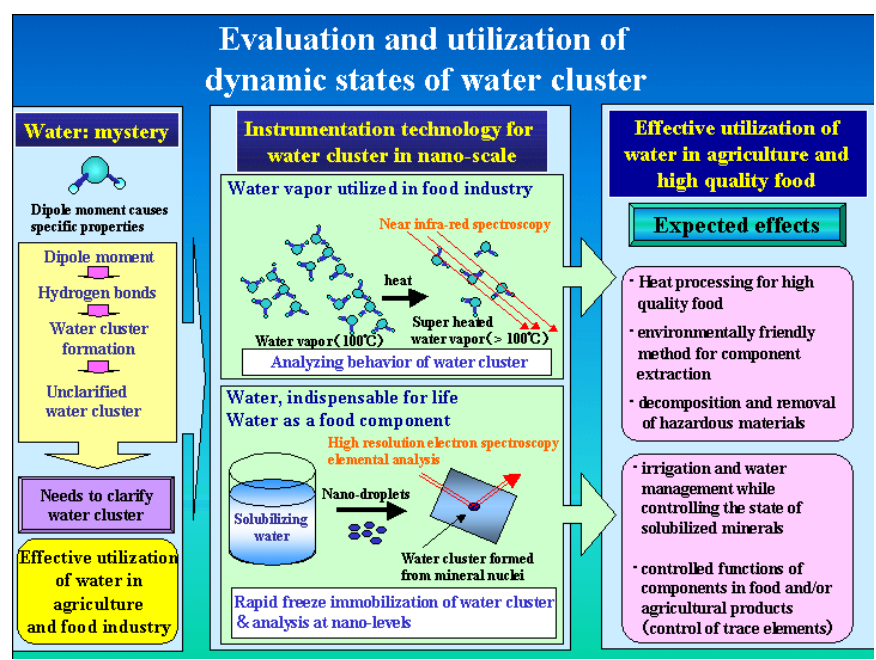

Fig. 11. Evaluation and utilization of dynamic states of water cluster

\section{Development of micro-bioreactor system}

Microreactor is equipped with a few 10 to a few $100 \mu \mathrm{m}$ sized microchannels that 
increase much the reaction efficiency, because the (surface or interface) reaction area per volume is extremely large and molecular diffusion is achieved very fast. MAFF started a new team for "mircobioreactor" in the research project on nanotechnology and materials since 2003. Kitamori, team leader, has investigated integrated chemistry lab, lab-on-a-chip, micro chemistry system, microchip, microfluidics, thermal lens microscope systems. At a national level, MAFF will be mainly in charge of utilization of biological molecules and functions in micro-reactor. The purpose of the team is to establish basic micro-bioreactor technology for cell culture, and the scheme is shown in Figure 12 [Kitamori, 2002; Kitamori, 2004; Yamamoto, 2002]. Main subjects are as follows. Development of a microchip-based bioreactor system for high throughput and highly efficient production of valuable materials such as realization of cell culture in a microspace with growth/differentiation control [Kitamori, 2004], development of micro breeder system including external fertilization, and efficient production of expensive and highly valuable materials by the biomicroreactor.

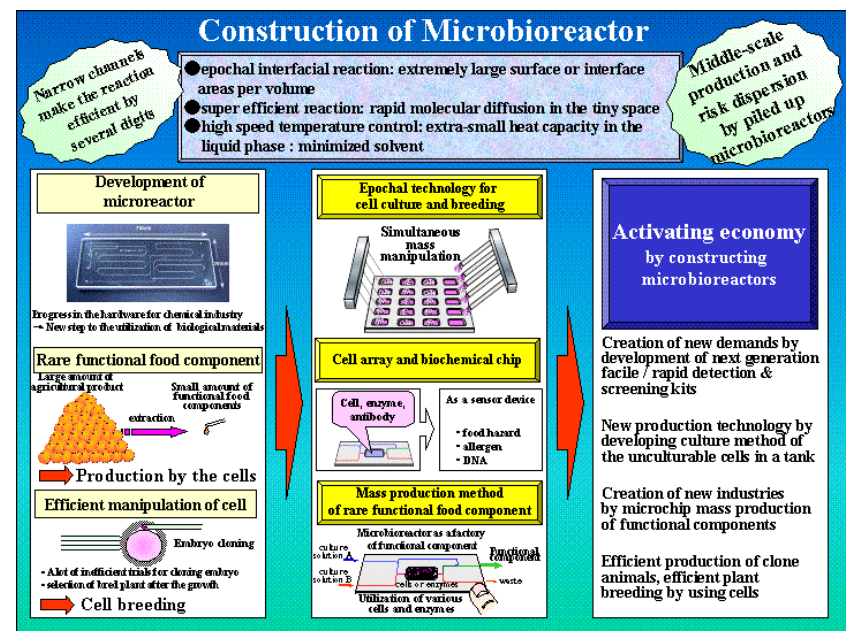

Fig. 12. Construction of microbioreactor.

\section{Acknowledgement}

All the above subjects have been conducted by the support of the Ministry of Agriculture, Forestry and Fisheries (MAFF) of Japan.

\section{Literatures cited}

Kikuchi, Y.; http://www.nfri.affrc.go.jp/guidance/soshiki/kikaku/microchanel.html

Kikuchi, H.E., Y. Kikuchi and Y. Kukoki; "Culture of bone-marrow-derived cells in microfabricated pit arrays," SPIE, 4265, 40-49 (2001).

http://www.kuraray.co.jp/release/2005/050629.html

Kobayashi, I., M. Nakajima, K. Chun, Y. Kikuchi and H. Fujita; "Silicon array of elongated through-holes for monodisperse emulsion droplets," AIChE J, 48(8), 1639-1644(2002)

Kobayashi I, Mukataka S, Nakajima M, Effects of type and physical properties of oil phase on oil-in-water emulsion droplet formation in straight-through microchannel emulsification, 
experimental and CFD Studies; Langmuir, 21, 5722-5730(2005)

Kasai W., Kondo T.; Fabrication of honeycomb patterned cellulose films, Macromol. Biosci., 4, 17-21 (2004)

Kitamori, T., Development of Microbioreactor, Proceedings of MAFF Nanotechnology

Symposium, p.81, in Japanese (2004)

Kondo, T.; http://ffpsc.agr.kyushu-u.ac.jp/pol/

Kondo, T., M. Nojiri, Y. Hishikawa, E. Togawa, D. Romanovicz and R. M. Brown Jr.; "Biodirected epitaxial nanodeposition of polymers on oriented macromolecular templates," Proceedings of National Academy of Science USA (PNAS), 99 (22), 14008-14013 (2002).

Kondo, T.; Proceedings of MAFF Nanotechnology Symposium, in Japanese, NFRI and AFFRC, Nov. 5, 2004, p.63-68

Nakajima, M.; "Development of microchannel emulsification technology for monodispersed droplets production,” Jap. J. Food Eng, 5, 71-81(2004)

Nakajima, M.; Proceedings of MAFF Nanotechnology Symposium, in Japanese, NFRI and AFFRC, Nov. 5, 2004, p.37-46

Nakanishi, T.M., Y. Okuni, Y. Hayashi and H. Nishiyama; "Water movement at bean plant roots by neutron beam analysis," J. Radioanal. Nucl. Chem. (2004).

Nakanishi, T.M..; Proceedings of MAFF Nanotechnology Symposium, in Japanese, NFRI and AFFRC, Nov. 5, 2004, p.69-80

Sugiura S., Oda T., Aoyagi Y., Matsuo R., Enomoto T., Matsumoto K, Namamura T., Satake M., Ochiai A., Ohkohchi N., Nakajima M., Microfabricated airflow nozzle for microencapsulation of living cells into 150 micrometer microcapsules: Biomedical Microdevices, accepted (2006)

Tamada, Y.;"Response of bilayer membrane containing the extracts of the labial palpus of fresh fly to taste stimuli," Biosensors 2002, May 2002, Kyoto.

Tamada, Y., Proceedings of MAFF Nanotechnology Symposium, p.47, in Japanese (2004)

Tamada, Y.; Proceedings of MAFF Nanotechnology Symposium, in Japanese, NFRI and AFFRC, Nov. 5, 2004, p.47-54

Tan C.P, Nakajima M, $\beta$-Carotene nanodispersions: preparation, characterization and stability evaluation; Food Chemistry, 92, 661-671(2005)

Tanaka, Y., K. Sato, M. Yamato, T. Okano and T. Kitamori; "Cell culture and life support system for microbioreactor and bioassay," Electrophoresis, (2004).

Yamamoto, K.;'MAFF research project on nanotechnology and materials, Proceedings of the United States-Japan Cooperatire Panel (UJNR) (2002)

Yazawa, M. and Kikuchi Y.; Proceedings of MAFF Nanotechnology Symposium, in Japanese, NFRI and AFFRC, Nov. 5, 2004, p.27-36 